\title{
Analyzing Electrocardiography Abnormalities in COVID-19 Patients Admitting to Emergency Department
}

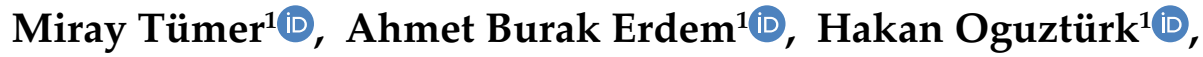 \\ Selahattin Gürü̈${ }^{1}$, Gülhan Kurtoglu Çelik ${ }^{1}$, Afsin Kayipmaz ${ }^{1}$ (D)
}

1 Ankara City Hospital, Department of Emergency Medicine, Ankara, Turkey

\begin{abstract}
Background: One of the groups affected at the highest level by the outbreak with the highest mortality rate is individuals with known cardiovascular diseases. Proinflammatory mediators play an important role in the pathophysiology of the disease cause cardiac and arrhythmic complications. This article aims to provide practical recommendations by evaluating ECG data in the follow-up and management of the disease to the Emergency Medicine workers to whom patients affected by COVID-19 first apply.

Methods: We randomly collected the ECG data of the patients applying to our Hospital Emergency Medicine Clinic green, yellow and red areas who were positive for viral RNA in throat swabs were carried out.

Results: The ECGs of 502 patients diagnosed with COVID-19 were collected in the 2-week period between June 15, 2020, and July 15, 2020. The study included 287 males $(57.17 \%)$ and 215 females (42.83\%). Normal sinus rhythm was detected in 241 patients $(48.01 \%)$ at ECG. A total of 3 ECG patterns, which suggested ST elevation, and 11 ECG patterns, which suggested ST depression, were decisive in terms of emergency intervention. The number of patients with atrial fibrillation was $17(3.39 \%)$.

Conclusions: This infection was associated with multiple, direct, and indirect cardiovascular complications, including acute myocardial damage, myocarditis, arrhythmias. Sinus tachycardia was determined to be $30.08 \%$, LVH 5.38\%, and VES $4.18 \%$ in our study. As a result, we believe that our initial ECG recommendation is that cardiac monitoring will play an important role in treatment planning.
\end{abstract}

Keywords: COVID-19, Electrocardiogram, Comorbidity, Emergency Department.

\section{INTRODUCTION}

After the COVID-19 disease, which is caused by the novel coronavirus (Severe Acute Respiratory Coronavirus-2 (SARS-CoV-2), which emerged in Wuhan, Hubei, China, in December 2019, and rapidly spread to nearly 200 countries around the world, was also detected and declared officially in our country on March 11, 2020, the number of cases has increased rapidly. One of the groups affected at the highest level by the outbreak with the highest mortality rate is individuals with known cardiovascular diseases (1). SARS-CoV-2 infection is associated with several proinflammatory diseases. Proinflammatory mediators play an important role in the pathophysiology of the 
disease cause cardiac and arrhythmic complications $(1,2)$. Electrocardiography (ECG) changes due to microvascular damage (ST elevation, non-specific ST-T segment changes, and rhythm disorders), rise in cardiac biomarkers (troponin and natriuretic peptide levels), and accompanying myocardium involvement can be detected in patients with respiratory distress and admitted to the emergency department due to COVID-19 (1-5).

This article aims to provide practical recommendations by evaluating ECG data in the follow-up and management of the disease to the Emergency Medicine workers to whom patients affected by COVID-19 first apply.

\section{MATERIALS AND METHODS}

Our hospital is a 3200- bed tertiary referral hospital in the central region of Turkey. This prospective study was conducted from June 15 to July 15, 2020, at the Emergency Department (ED) of our hospital. The current study was approved by the Ankara City Hospitals' Ethics committee (Date: 21.05.2020, No: E1-20-599), and permissions were obtained. After ethical approval, we randomly collected the ECG data of the patients applying to our Hospital Emergency Medicine Clinic to the green, yellow, and red areas who were positive for viral RNA in throat swabs were carried out.

Patients who were at and above the age of 18 wereincluded in the study. The study was conducted 24 hours, seven days a week. After patients' admission to the emergency department with COVID-19 symptoms, throat swap was collected for viral RNA. Positive patients' ECG data were collected and loaded to the SPSS database. All the ECG results were evaluated by an emergency medicine specialist who was also an ECG trainer. Continuous data are expressed as mean standard deviation (SD) and categorical data as number (percentage). All statistical analyses were performed using SPSS version 26.0 (IBM Corporation, Armonk, NY).

\section{RESULTS}

The ECGs of 502 patients diagnosed with COVID-19 were collected in the 2-week period between June 15, 2020, and July 15, 2020. The study included 287 males $(57.17 \%)$ and 215 females (42.83\%). The mean age was $50.45 \pm 19.14$ years (18-98 years).

Normal sinus rhythm was detected in 241 patients $(48.01 \%)$ at ECG. As a result of ECG examinations of the patient, many rhythm disorders were detected in the remaining $261(51.99 \%)$. A total of 3 ECG patterns, which suggested ST elevation, and 11 ECG patterns, which suggested ST depression, were decisive in terms of emergency intervention. The number of patients with atrial fibrillation was 17 (3.39\%). As a result of the followups of 502 patients in the Emergency Department: 1 patient died in the Emergency Department, 142 (28.29\%) patients were admitted to the intensive care unit, and $246(22.5 \%)$ patients were admitted to isolated COVID services. The other $263(44.42 \%)$ patients who had COVID-19 positive but without symptoms and complaints were guided to isolated accommodation with medical advice accompanied by paramedics. Three patients were admitted to the Cardiology Coronary Care Unit for invasive intervention and follow-up purposes. The ECG of the patient who died in the Emergency Department was compatible with inferior MI.

Table 1. Emergency Department Admittance of COVID-19 Patients: Electrocardiography Evaluations (n: 502)

\begin{tabular}{|l|l|l|}
\hline Variable & $\mathbf{n}$ & $\mathbf{( \% )}$ \\
\hline Sinus rhythm & $\mathbf{2 4 1}$ & 48.01 \\
\hline Sinus tachycardia & $\mathbf{1 5 1}$ & 30.08 \\
\hline Sinus bradycardia & $\mathbf{1 4}$ & 2.79 \\
\hline ST elevation & $\mathbf{3}$ & 0.60 \\
\hline RHV & $\mathbf{1 9}$ & 3.78 \\
\hline LVH & $\mathbf{2 7}$ & 5.38 \\
\hline Atrial fibrillation & $\mathbf{1 7}$ & 3.39 \\
\hline LBBB & $\mathbf{8}$ & 1.59 \\
\hline RBBB & $\mathbf{1 2}$ & 2.39 \\
\hline Ventricular early systole & $\mathbf{2 1}$ & 4.18 \\
\hline Anterior ischemia & $\mathbf{2}$ & 0.40 \\
\hline Inferior ischemia & $\mathbf{1 1}$ & 2.19 \\
\hline AV Block $1^{0}$ & $\mathbf{2}$ & 0.40 \\
\hline QT extension & $\mathbf{0}$ & 0.00 \\
\hline
\end{tabular}

LVH: Left Ventricular Hypertrophy, RVH: Left Ventricular Hypertrophy LBBB: Left bundle branch block, RBBB: Right bundle branch block

The three most common diseases in the comorbidities of the patients were hypertension in 127 patients $(25.30 \%)$, cardiovascular disease in 112 patients $(22.31 \%)$, Diabetes Mellitus in 109 patients $(21.71 \%)$. The top three symptoms were weakness in $51.00 \%$ of patients, cough in $50.60 \%$, fever in $42.03 \%$, respectively. 
Table 2. Comorbidities in the Cases

\begin{tabular}{|l|c|c|}
\hline Comorbidities & $\mathbf{n}$ & $(\boldsymbol{\%})$ \\
\hline Diabetes Mellitus & 109 & 21.71 \\
\hline Hypertension & 127 & 25.30 \\
\hline Cardiovascular Disease & 112 & 22.31 \\
\hline Chronic Lung Disease & 106 & 21.12 \\
\hline Chronic Kidney Disease & 33 & 6.57 \\
\hline Chronic Liver Disease & 47 & 9.36 \\
\hline Malignancy & 74 & 14.74 \\
\hline Cerebrovascular Disease & 68 & 13.55 \\
\hline
\end{tabular}

Table 3. Frequency of Symptoms

\begin{tabular}{|c|c|c|}
\hline Symptoms & $\mathrm{n}$ & $\%$ \\
\hline Chest Pain & 116 & 23.11 \\
\hline Fever & 211 & 42.03 \\
\hline Cough & 254 & 50.60 \\
\hline Palpitation & 102 & 20.32 \\
\hline Shortness of Breath & 186 & 37.05 \\
\hline Weakness & 256 & 51.00 \\
\hline Headache & 165 & 32.87 \\
\hline Anosmia & 9 & 1.79 \\
\hline Ageusia & 12 & 2.39 \\
\hline Diarrhea & 24 & 4.78 \\
\hline Asymptomatic & 223 & 44.42 \\
\hline
\end{tabular}

\section{DISCUSSION}

The main characteristics of SARS-CoV-2, the new member of the Coronavirus family, are that it easily binds especially to lung Type 2 alveoli cells, ACE2 receptor in myocardia, kidney proximal tubules esophagus, intestinal epithelial cells, and bladder urothelial cells in humans (7). COVID-19, which has a high infectious characteristic, is spread especially through droplets and direct contact. The average incubation period is 5.5 days; however, it is also known to last up to 14 days (5). The symptoms of the disease usually appear within the first 14 days. In symptomatic cases, the most common symptom is fever, dry cough, weakness, muscle pain, sore throat, less frequent nauseavomiting, and diarrhea (9). Although it is reported in the literature that the most frequent and higher rate symptom was fever in COVID-19 patients with lung infection at a rate of $31-89 \%(9,10)$, the most common complaint was weakness in our study at a rate of $51.00 \%$, and high fever was $42.03 \%$ ranking the $3^{\text {rd }}$ frequent symptom. It is also important to question and follow-up on the ageusia and anosmia disorders and diarrhea complaints reported in the literature in COVID 19 patients (11-13). As a matter of fact, $4.78 \%$ of the patients who were admitted to our Emergency Department had diarrhea, $1.79 \%$ were unable to smell, and $2.39 \%$ and $2.39 \%$ complained that they could not taste.

Patients with underlying medical problems like the elderly, hypertension, cardiovascular disease, or diabetes are more likely to develop serious diseases. Individuals from all age groups may be infected with COVID-19, but adults of middle age and older are more frequently affected and are more likely to experience the disease more severely. In the literature, the median age of patients diagnosed with COVID-19 in hospitals ranges between 49 and 56 (9-11). In our study, the mean age was $50.45 \pm 19.14$ years.

The COVID-19 outbreak presented several difficulties in terms of cardiovascular emergencies. The risk of disease severity and death increased in COVID-19 patients with previous cardiovascular disease. This infection was associated with multiple, direct, and indirect cardiovascular complications, including acute myocardial damage, myocarditis, arrhythmias, and venous thromboembolism. The most important characteristic that makes COVID-19 infection so dreadful is that it causes respiratory failure, especially in high-risk cases; and it can severely disrupt the cardiac hemodynamic with direct myocardial development. ECG changes (ST elevation and non-specific ST-T segment changes) and elevation in cardiac biomarkers (i.e., troponin, etc.) are detected in COVID-19 patients with respiratory distress admitting to Emergency Department. It was reported in a previously published patient series that $40 \%$ of patients admitted due to COVID-19 positivity had cardiovascular or cerebrovascular diseases, $17 \%$ might develop arrhythmia, and 7\% might develop acute cardiac damage. In a case reported, however, it was reported that the initial admission in COVID-19 might be acute-onset heart failure, acute Myocardial Infarction (ME), myocarditis, and sudden cardiac arrest $(14,17,18)$. The cardiovascular disease rate in terms of comorbidity was reported between $14.5 \%$ and $40 \%$, hypertension $14.6 \%$ and $31.2 \%$, and diabetes 10.1 and $19.5 \%$ in some publications (9). In our cases, in line with the literature, the cardiovascular rate was $22.31 \%$, hypertension $25.30 \%$, and diabetes $21.71 \%$.

The clinical impact of COVID-19 infection will be greater with advanced age and comorbidities. In this sense, it was reported that previously cardiovascular patients accounted for $22.7 \%$ of all the morbidities (19). Most 
patients suspected of COVID-19 and especially patients with severe diseases or those with the probability of QTextension should undergo ECG after admitting to the Emergency Department (20); ideally, this may be a 12-lead ECG (21). This will allow that the initial QRS-T morphology is documented in case symptoms develop, suggesting myocardial damage or acute coronary syndrome in the patient. In our study, we find no QT extension patients.

There are no adequate publications in the literature about cardiac rhythm disorders in COVID 19 patients. Arrhythmias were reported in 17\% of patients and in $44 \%$ of patients admitted to the Intensive Care Unit in a study conducted with a total of 138 patients with pneumonia associated with Wuhan-induced COVID-19 (14). It was reported in another study that atrial arrhythmias were more common in patients requiring mechanical ventilation (22), and the frequency of ventricular tachyarrhythmias was also mentioned (23). There were T-wave depression and inversion, ST-segment elevation or depression, and $\mathrm{Q}$ waves among the coronary ECG abnormalities reported in patients with COVID-19 (24-27). In case of ST-segment elevation, other diagnostic examinations are necessary to distinguish MI from other causes of myocardial damage (25-27). In a series that included 18 COVID-19 patients with ST-segment elevation, MI developed clinically due to coronary obstruction in 8 patients (coronary angiographies that confirmed obstructive coronary disease were carried out in 6), and the remaining 10 patients were diagnosed with non-coronary myocardial damage (27), 4 of the patients with MI and 9 of those with non-coronary myocardial damage died in hospital. Obstruction was confirmed with Coronary Angiography in two of our three patients with ST-elevation, and one died despite the advanced cardiac life support.

The most common arrhythmia in COVID-19 patients is sinus tachycardia; however, atrial fibrillation, atrial flutter or ventricular tachycardia might also occur (27). Sinus tachycardia was determined to be $30.08 \%, \mathrm{LVH} 5.38 \%$, and VES $4.18 \%$ in our study.

An important issue that should not be ignored in this process may be that physicians might focus on symptoms and complaints of this disease because of primary COVID 19 diseases and neglect the presence of cardiac problems, incomplete diagnosis, or delayed diagnosis. A significant problem in MI patients who require medical care may also occur for emergencies where admissions of patients are made separately because of delays in diagnoses and treatments. Since the delay of specific treatments of acute coronary problems can cause cardiovascular emergencies, standardization is also needed for interventional cardiology.

Our study showed that ECG plays an important role in diagnosing, following up, and treating patients admitted with COVID 19 pre-diagnosis. There are several limitations to consider when interpreting the results of our study. Firstly, the study duration was short (1 month), and the study included only one hospital; for this reason, more studies are needed to generalize the results.

It is necessary to determine the treatment process by considering cardiovascular complications and non-COVID emergencies for COVID-19 patients. Continuous ECG monitoring is not required for the patient if the cardiac arrhythmias are documented if there are no suspicious myocardial ischemia or other standard indications. However, as part of the infection control mechanisms for patients with or suspected COVID-19, continuous ECG follow-up by many hospital nurses instead of standard vital parameter follow-ups requires that clinical staff are also careful about the risk of infection.

Emergency Medicine workers must be cautious and consider the intense distribution of ACE2, which is the binding point of SARS-CoV-2, in cardiomyocytes and monitor changes in the ECG in the Emergency Department step and see the problems. As a result, we believe that our initial ECG recommendation and cardiac monitoring will play important roles in treatment planning.

\section{Declarations}

The authors received no financial support for the research and/or authorship of this article. There is no conflict of interest.

The current study was approved by the Ankara City Hospitals' Ethics committee (Date: 21.05.2020, No: E1-20-599), and permissions were obtained. 


\section{REFERENCES}

1- American College of Cardiology. Troponin and BNP use in COVID-19. Available at: https://www.acc.org/latest-in-cardiology/ articles / 2020/03/18/15/25/troponin-and-bnp-use-in-covid19?utm_ source $=$ accupdate\&utm_medium $=$ email_newsletter\&utm _ content=20200320\&utm_campaign=accupdate. Accessed March 23, 2020

2- Azevedo RB, Botelho BG, Hollanda JVG, Ferreira LVL, Junqueira de Andrade LZ, et al. Covid-19 and the cardiovascular system: a comprehensive review. J Hum Hypertens. 2021;35(1):4-11.

3- Kochi AN, Tagliari AP, Forleo GB, Fassini GM, Tondo C. Cardiac and arrhythmic complications in patients with COVID-19. J Cardiovasc Electrophysiol. 2020;31(5):1003-1008.

4- Jankelson L, Karam G, Becker ML, Chinitz LA, Tsai MC. QT prolongation, torsades de pointes, and sudden death with short courses of chloroquine or hydroxychloroquine as used in COVID-19: A systematic review. Heart Rhythm. 2020;17(9):1472-1479.

5- Chorin E, Wadhwani L, Magnani S, Dai M, Shulman E, NadeauRouthier C, et al. QT interval prolongation and torsade de pointes in patients with COVID-19 treated with hydroxychloroquine/ azithromycin. Heart Rhythm. 2020;17(9):1425-1433.

6- van den Broek MPH, Möhlmann JE, Abeln BGS, Liebregts M, van Dijk VF, van de Garde EMW. Chloroquine-induced QTc prolongation in COVID-19 patients. Neth Heart J. 2020;28(7-8):406-409.

7- Andersen KG, Rambaut A, Lipkin WI, Holmes EC, Garry RF. The proximal origin of SARS-CoV-2. Nat Med. 2020;26(4):450-452.

8- Lauer SA, Grantz KH, Bi Q, Jones FK, Zheng Q, Meredith HR, Azman AS, Reich NG, Lessler J. The incubation period of Coronavirus disease 2019 (COVID-19) from publicly reported confirmed cases: Estimation and application. Ann Intern Med. 2020;172(9):577-582.

9- Guan WJ, Ni ZY, Hu Y, Liang WH, Ou CQ, He JX, et al. Clinical characteristics of Coronavirus disease 2019 in China. N Engl J Med. 2020;382(18):1708-1720.

10- Richardson S, Hirsch JS, Narasimhan M, Crawford JM, McGinn T, Davidson KW, et al. Presenting characteristics, comorbidities, and outcomes among 5700 patients hospitalized with COVID-19 in the New York City area. JAMA. 2020;323(20):2052-2059.

11- Sutton D, Fuchs K, D'Alton M, Goffman D. Universal screening for SARS-CoV-2 in women admitted for delivery. N Engl J Med. 2020; 382:2163

12- Arons MM, Hatfield KM, Reddy SC, Kimball A, James A, Jacobs JR, et al. Presymptomatic SARS-CoV-2 infections and transmission in a skilled nursing facility. N Engl J Med. 2020;382(22):2081-2090.

13- Campbell KH, Tornatore JM, Lawrence KE, Illuzzi JL, Sussman LS, Lipkind HS, et al. Prevalence of SARS-CoV-2 among patients admitted for childbirth in southern Connecticut. JAMA. 2020;323(24):2520-2522.

14- Wang D, Hu B, Hu C, Zhu F, Liu X, Zhang J, et al. Clinical Characteristics of 138 Hospitalized Patients With 2019 Novel Coronavirus-Infected Pneumonia in Wuhan, China. JAMA. 2020;323(11):1061-1069.

15- Chen N, Zhou M, Dong X, Qu J, Gong F, Han Y, et al. Epidemiological and clinical characteristics of 99 cases of 2019 novel coronavirus pneumonia in Wuhan, China: a descriptive study. Lancet. 2020;395(10223):507-513.

16- Liu K, Fang YY, Deng Y, Liu W, Wang MF, Ma JP, et al. Clinical characteristics of novel coronavirus cases in tertiary hospitals in Hubei Province. Chin Med J (Engl). 2020;133(9):1025-1031.

17- Huang C, Wang Y, Li X, Ren L, Zhao J, Hu Y, et al. Clinical features of patients infected with 2019 novel coronavirus in Wuhan, China. Lancet. 2020;395(10223):497-506.
18- Zeng J, Huang J, Pan L. How to balance acute myocardial infarction and COVID-19: the protocols from Sichuan Provincial People's Hospital. Intensive Care Med. 2020;46(6):1111-1113.

19- Erganiş S, Bozdayı G. Virology, immunity and vaccine development of SARS-CoV-2. Arch Curr Med Res 2021;2(1):05-13

20- Gandhi RT, Lynch JB, Del Rio C. Mild or moderate Covid-19. N Engl J Med. 2020;383(18):1757-1766.

21- Cheung CC, Davies B, Gibbs K, Laksman ZW, Krahn AD. Multilead QT Screening is decessary for QT measurement: Implications for management of patients in the COVID-19 era. JACC Clin Electrophysiol. 2020;6(7):878-880.

22- Ceyhan MA, Tümer M, Gürü S, Kadı G, Demir GG. Mean platelet volume and red cell distribution width values in patients with COVID-19 admitted to intensive care units or wards from emergency department. Arch Curr Med Res. 2021;2(2):88-92.

23- Guo T, Fan Y, Chen M, Wu X, Zhang L, He T, et al. Cardiovascular implications of fatal outcomes of patients with Coronavirus disease 2019 (COVID-19). JAMA Cardiol. 2020;5(7):811-818.

24- Shi S, Qin M, Shen B, Cai Y, Liu T, Yang F, et al. Association of cardiac injury with mortality in hospitalized patients with COVID-19 in Wuhan, China. JAMA Cardiol. 2020;5(7):802-810.

25- Inciardi RM, Lupi L, Zaccone G, Italia L, Raffo M, Tomasoni D, et al. Cardiac involvement in a patient with Coronavirus disease 2019 (COVID-19). JAMA Cardiol. 2020;5(7):819-824.

26- $\mathrm{Hu} \mathrm{H}$, Ma F, Wei X, Fang Y. Coronavirus fulminant myocarditis treated with glucocorticoid and human immunoglobulin. Eur Heart J. 2021;42(2):206.

27- Bangalore S, Sharma A, Slotwiner A, Yatskar L, Harari R, Shah B, et al. ST-segment elevation in patients with Covid-19 - A case series. N Engl J Med. 2020;382(25):2478-2480. 